\title{
Neue Beschäftigungsformen in Europa
}

\author{
Irene Mandl
}

\section{Neue Beschäftigungsformen in Europa- ein Definitionsansatz}

Die traditionelle Standardbeschäftigung (unbefristeter Vollzeitarbeitsvertrag oder Selbstständigkeit) ist nach wie vor die dominante Beschäftigungsform in Europa. Innerhalb der letzten zehn bis fünfzehn Jahre zeigt sich jedoch eine steigende Vielfalt an Beschäftigungsformen. Dieser Trend ist neben den durch die Wirtschaftskrise ausgelösten kürzlichen Entwicklungen am Arbeitsmarkt auch auf allgemeinere Faktoren wie die steigende Bedeutung bestimmter Branchen und Berufe, den vermehrten Einsatz moderner Informations- und Kommunikationstechnologien, gesellschaftliche Entwicklungen oder den allgemeinen Wunsch vieler Arbeitgeber und Arbeitnehmerlnnen nach höherer Flexibilität zurückzuführen.

Obwohl die aktuelle Arbeitsmarktsituation, beobachtbare Trends und Zukunftsszenarien vielfach Thema der politischen und öffentlichen Diskussion sind, gibt es bislang wenig konkrete Informationen über die neuen Beschäftigungsformen in Europa und deren Auswirkungen auf den Arbeitsmarkt und die Arbeitsbedingungen der betroffenen Arbeitnehmerlnnen. Um zur Schließung dieser Wissenslücke beizutragen, führte die Europäische Stiftung zur Verbesserung der Lebens- und Arbeitsbedingungen (Eurofound) in 2013/2014 eine Studie durch, ${ }^{1}$ deren Ergebnisse im Folgenden zusammengefasst werden.

In diesem Beitrag werden „neue Beschäftigungsformen“ als jene Trends am Arbeitsmarkt verstanden, die seit ca. 2000 neu oder in verstärktem Ausmaß zu beobachten sind. Dabei wurde ein nationaler Ansatz gewählt: Was in einem Mitgliedsstaat der Europäischen Union neu ist, kann in einem anderen bereits lange etabliert sein und in einem dritten Land (noch) gar nicht auftreten. Diskutiert werden jene Beschäftigungsformen, die in mehreren Ländern als neu identifiziert wurden.

Neue Beschäftigungsformen - wie im gegenwärtigen Beitrag verstanden - können die folgenden Ausprägungen (einzeln oder in Kombination) haben:

- Arbeitsbeziehungen, die vom traditionellen Konzept „ein Arbeitgeber - ein/e Arbeitnehmerln" abweichen, indem entweder eine Gruppe von Arbeitgebern gemeinsam eine/n Mitarbeiterln beschäftigt oder ein 
Arbeitgeber für einen bestimmten Arbeitsplatz mehrere Mitarbeiterlnnen anstellt, die gemeinsam die Aufgaben erfüllen. Leiharbeit bzw. Personalbereitstellung wurde in diesem Zusammenhang nicht als „neu“ betrachtet.

- Arbeitsbeziehungen, die diskontinuierlich bzw. sporadisch oder von sehr kurzer Dauer sind. Etablierte Modelle der Teilzeit- oder Saisonarbeit wurden nicht berücksichtigt.

- Kooperationen zwischen Selbstständigen, die über die klassischen Beziehungen entlang der Wertschöpfungskette, gemeinsame Projektarbeit oder gemeinsame Räumlichkeiten hinausgehen.

- Arbeitsplätze, die von den Räumlichkeiten des Arbeit- oder Auftraggebers abweichen, insbesondere "mobile Tätigkeiten“ ohne festen Arbeitsort. Telearbeit wurde nicht als "neu“ berücksichtigt.

- Tätigkeiten, die stark von modernen Informations- und Kommunikationstechnologien abhängen und bei denen die Anwendung dieser Technologien die Natur der Arbeitsbeziehungen, Arbeitsinhalte oder Arbeitsorganisation verändert.

Berücksichtigt wurden sowohl Beschäftigungsformen, die für die Gesamtwirtschaft relevant sind, als auch solche, die lediglich für bestimmte Branchen, Berufe oder Tätigkeiten Anwendung finden. In ähnlicher Weise wurden sowohl Arbeitsverhältnisse untersucht, für die spezifische gesetzliche oder kollektivvertragliche Regelungen vorliegen, solche, die dem allgemeinen Arbeitsrecht unterliegen, und jene, die gänzlich ungeregelt sind.

\section{Abbildung 1: Definition neuer Beschäftigungsformen in Europa}

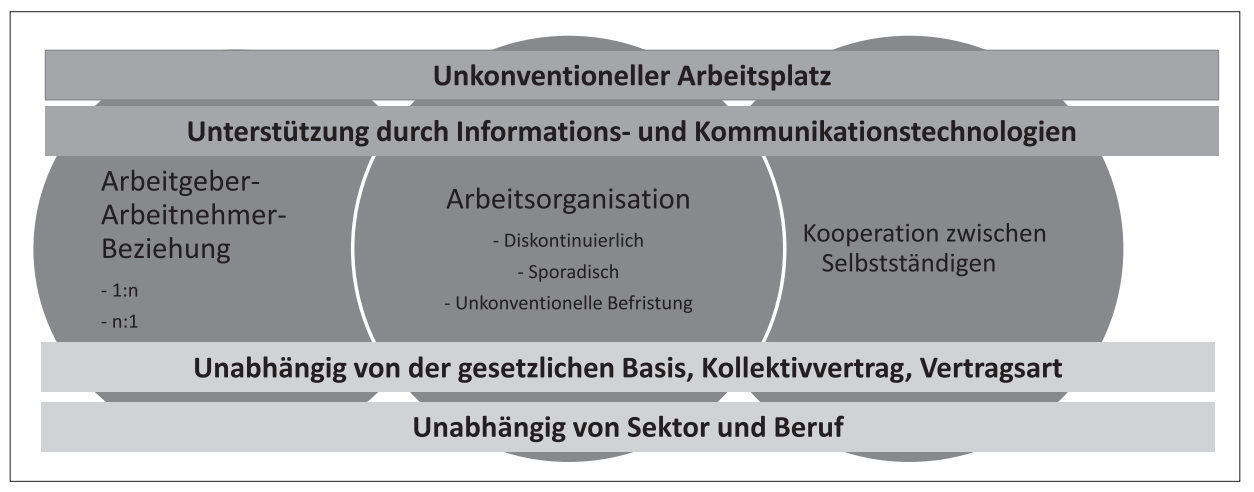

Quelle: Eurofound (2015).

Basierend auf dieser Definition wurden neun neue Beschäftigungstrends in Europa identifiziert, die im Folgenden kurz beschrieben werden. Dabei ist anzumerken, dass die neun Kategorien sich nicht unbedingt gegenseitig ausschließen, sondern überlappen können, $d$. h. ein konkretes 
Beschäftigungsverhältnis kann in mehr als eine der anschließend vorgestellten Kategorien fallen.

\section{Mitarbeitersharing (Arbeitgeberzusammenschlüsse)}

\subsection{Allgemeine Charakteristika}

Beim Mitarbeitersharing wird ein/e Mitarbeiterln gemeinschaftlich von einer Gruppe von Arbeitgebern beschäftigt, die ihre planbaren, aber fragmentierten Personalbedarfe bündeln und so kooperativ einen unbefristeten Vollzeitarbeitsplatz schaffen. Die beteiligten Unternehmen gründen einen sogenannten Arbeitgeberzusammenschluss (AGZ), der die Personalplanung und -koordination übernimmt. Der AGZ wird der formale Arbeitgeber der Arbeitskräfte und stellt deren Dienste den beteiligten Unternehmen in Rechnung. Die einzelnen Unternehmen sind für die Arbeitsorganisation in ihren jeweiligen Betrieben zuständig.

Ähnlich wie bei der Leiharbeit/Personalbereitstellung arbeiten die MitarbeiterInnen somit operativ in verschiedenen Betrieben. Allerdings wissen sie bereits bei der Anstellung, in welche Unternehmen sie entsandt werden, und kehren in regelmäßigen Abständen in den gleichen Betrieb zurück. Der AGZ ist nicht darauf ausgelegt, aus der Koordination der Arbeitskräfte Gewinn zu erwirtschaften und ist durch eine gemeinschaftliche Verantwortung der beteiligten Betriebe, die Auslastung der Mitarbeiterlnnen zu gewährleisten, gekennzeichnet.

Arbeitgeberzusammenschlüsse sind insbesondere für die folgenden Tätigkeiten geeignet: ${ }^{2}$

- Saisonarbeit, die mit antizyklischen Tätigkeiten kombiniert wird,

- Kombination verschiedener Teilzeittätigkeiten (z. B. im Einzelhandel oder Tourismus),

- spezialisierte Tätigkeiten, für die z. B. kleine und mittlere Unternehmen (KMU) Personalbedarf haben, aber nicht in Vollzeitausmaß (z. B. Marketing, EDV),

- strategische Projekte, für deren Entwicklung insbesondere in KMU keine ausreichenden Personalressourcen zur Verfügung stehen.

Sie können in allen Branchen Anwendung finden, wobei in der Praxis eine Kombination verschiedener Wirtschaftszweige empfehlenswert ist, um eine bessere kontinuierliche Auslastung der Mitarbeiterlnnen zu gewährleisten. Unternehmen aller Größenklassen können an Arbeitgeberzusammenschlüssen beteiligt sein, wobei sich diese Beschäftigungsform in erster Linie für KMU als vorteilhaft zeigt. Hauptmotivation für Unternehmen, sich an AGZ zu beteiligen, ist der Bedarf nach bestimmten Arbeitskräften, die jedoch nicht permanent bzw. in einer Vollzeitanstellung ausgelastet werden können. AGZ bieten die Möglichkeit, derartige Bedarfe zeit- 
und kostengünstig zu decken und, da die Mitarbeiterlnnen wiederholt eingesetzt werden, eine gewisse Personalkontinuität zu realisieren.

\section{Abbildung 2: Arbeitsweise von Mitarbeitersharing (Arbeitgeber- zusammenschlüssen)}

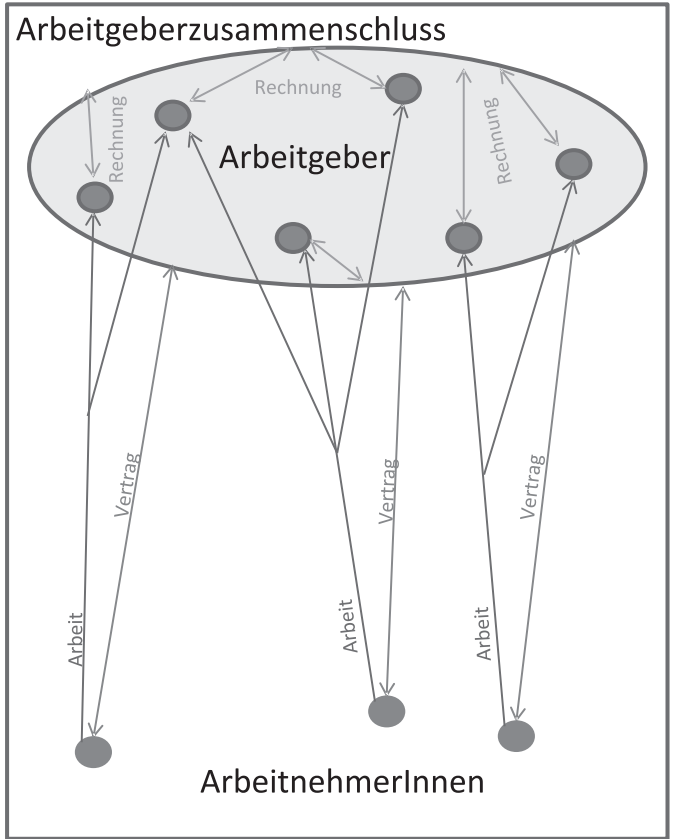

Quelle: Eurofound (2015).

ArbeitnehmerInnen in Arbeitgeberzusammenschlüssen können ein breites Spektrum an Berufen und Qualifikationsniveaus umfassen, von GeneralistInnen bis zu Spezialisten und von Niedrig- zu Hochqualifizierten, je nach Bedarf der beteiligten Unternehmen.

\subsection{Auswirkungen auf die Arbeitsbedingungen und den Arbeitsmarkt}

Arbeitgeberzusammenschlüsse tragen zur Beschäftigungsstabilität bei, da durch die Kombination der Personalbedarfe einzelner Unternehmen permanente Vollzeitbeschäftigung geschaffen wird, die andernfalls durch potenziell prekärere Arbeitsverhältnisse oder Arbeitsintensivierung der Kernbelegschaft gedeckt würden. ${ }^{3}$ Darüber hinaus wird den ArbeitnehmerInnen die Verhandlung der Arbeitsbedingungen erleichtert, da sie lediglich mit einem formellen Arbeitgeber konfrontiert sind, obwohl sie operativ in unterschiedlichen Betrieben arbeiten. ${ }^{4}$ Der Einsatz in verschiedenen Unternehmen erfordert von den MitarbeiterInnen allerdings ein gewisses 
Maß an Flexibilität und Anpassungsfähigkeit, inklusive der Bereitschaft, zu den einzelnen Betrieben zu pendeln.

Die AGZ-MitarbeiterInnen profitieren im Allgemeinen von den gleichen Arbeitsbedingungen wie die Kernbelegschaft der beteiligten Unternehmen, und teilweise ist eine Verbesserung der Arbeitsbedingungen in der Region zu beobachten, da eine Vereinheitlichung der Konditionen in den beteiligten Unternehmen zu beobachten ist. Dennoch kann es aufgrund der Unterschiede zwischen den Betrieben zu Schwankungen, z. B. hinsichtlich der Gehälter, Arbeitszeiten oder Arbeitsorganisation, kommen.

AGZ können zur Weiterentwicklung der Arbeitskräfte beitragen, da die MitarbeiterInnen in den unterschiedlichen Unternehmen unterschiedliche Erfahrungen sammeln und dadurch Kompetenz aufbauen können. Gleichzeitig birgt der regelmäßige Wechsel zwischen den Unternehmen die Gefahr, dass die Mitarbeiterlnnen sich durch die variierenden Aufgaben und Organisationsstrukturen gestresst fühlen, nicht vollständig in die Belegschaft eingebunden werden und ihre Anliegen von den Belegschaftsvertreterlnnen nur bedingt berücksichtigt werden können.

Für die beteiligten Unternehmen bieten AGZ Zugang zu Arbeitskräften, den sie andernfalls nicht oder mit höheren Kosten hätten. ${ }^{5}$ Die Betriebe profitieren auch von der überbetrieblichen Arbeitserfahrung der MitarbeiterInnen, die zu Effizienz- und Produktivitätsgewinnen führen kann. Darüber hinaus gibt es Hinweise, dass AGZ zur regionalen Kooperation zwischen Unternehmen beiträgt, die über die gemeinsame Personalpolitik hinausgeht.

\section{Jobsharing}

\subsection{Allgemeine Charakteristika}

Beim Jobsharing beschäftigt ein Arbeitgeber mehrere Arbeitnehmerlnnen (im Allgemeinen jedoch nur zwei), um gemeinsam einen bestimmten Vollzeitarbeitsplatz zu füllen. Es handelt sich somit um eine spezielle Form der Teilzeitarbeit, die den Bedarf der MitarbeiterInnen nach einer Teilzeitposition mit der Anforderung des Unternehmens, den Arbeitsplatz kontinuierlich zu besetzen und die gesamte Arbeitsplatzbeschreibung abzudecken, verbindet.

Während in manchen europäischen Ländern Jobsharing auf Basis des allgemeinen Arbeitsrechts (somit als bilaterale Vereinbarung zwischen dem Arbeitgeber und den Arbeitnehmerlnnen) realisiert wird, gibt es in anderen Mitgliedsstaaten der EU konkrete gesetzliche oder kollektivvertragliche Regelungen zu dieser Beschäftigungsform. Auch in diesen Fällen obliegt die operative Ausgestaltung jedoch den Arbeitgebern und MitarbeiterInnen. So kann die Arbeit entweder anhand der Arbeitszeit (z. B. arbei- 
tet ein/e Mitarbeiterln vormittags, der/die andere nachmittags) oder anhand der Aufgaben (z. B. übernimmt ein/e Mitarbeiterln den Kundenkontakt, der/die andere die internen administrativen Tätigkeiten) geteilt werden. Im manchen Fällen legt der/die Vorgesetzte eindeutig fest, welche/r Mitarbeiterln welche Aufgaben zu erfüllen hat, während in anderen Fällen lediglich das „Aufgabenpaket" an die MitarbeiterInnen übergeben wird, die sich untereinander einigen, wer welche Aufgabe erfüllt. Auch die Kommunikationspraktiken zwischen Vorgesetzten und den MitarbeiterInnen untereinander variieren von Fall zu Fall. Allerdings werden ein extensiver und kontinierlicher Kommunikationsfluss zwischen dem Arbeitgeber und den beteiligten Arbeitnehmerlnnen und gut durchdachte Strategien zur Übergabe der Aufgaben zwischen den Mitarbeiterlnnen als wesentliche Erfolgsfaktoren für Jobsharing hervorgehoben. ${ }^{6}$

Charakteristisch für die meisten Jobsharing-Modelle ist, dass die Mitarbeiterlnnen einander ersetzen müssen, z. B. falls eine der Arbeitskräfte durch Krankheit ausfällt. Somit wird - im Vergleich zu anderen Teilzeitmodellen - ein gewisser Bereich der klassischen Arbeitgeberverantwortung an die ArbeitnehmerInnen übertragen.

Jobsharing ist sowohl für niedrig als auch für hoch qualifizierte MitarbeiterInnen anwendbar, sofern die Aufgaben entsprechend teilbar sind und ein adäquater Jobsharing-Partner gefunden werden kann. ${ }^{7}$ Die wenigen verfügbaren Daten weisen darauf hin, dass Jobsharing aktuell von Frauen mit Kinderbetreuungspflichten dominiert ist und insbesondere im öffentlichen Sektor Anwendung findet.

\subsection{Auswirkungen auf die Arbeitsbedingungen und den Arbeitsmarkt}

Jobsharing ist eine flexible Beschäftigungsform, die Arbeitnehmerlnnen hilft, die von innen gewünschte Work-Life-Balance zu erzielen, und vielfach mit einem besseren Status und Karriereaussichten verbunden ist als "normale" Teilzeitarbeit. ${ }^{8}$ Durch die Möglichkeit des Austausches mit und Lernen von den Jobsharing-Partnern können Lerneffekte realisiert werden, die für die persönliche und professionelle Weiterentwicklung der betroffenen Mitarbeiterlnnen positiv sind.

Unzureichend organisierte bzw. implementierte Arbeitspraktiken und Kommunikationsstrategien können allerdings zu Konflikten zwischen den Beteiligten führen, ${ }^{9}$ und/oder es kann zu einer Arbeitsintensivierung, mehr Überstunden und arbeitsbezogenem Stress kommen. ${ }^{10}$

Aus makroökonomischer Sicht hat Jobsharing das Potenzial, zur Arbeitsmarktintegration benachteiligter Gruppen beizutragen, insbesondere von Personen mit Betreuungspflichten oder jenen, die wegen gesundheitlicher Gründe keine Vollzeitarbeit annehmen können. 
Darüber hinaus könnte Jobsharing auch als Instrument zur Bekämpfung der Jugendarbeitslosigkeit eingesetzt werden, indem Modelle etabliert werden, in denen ältere Arbeitnehmerlnnen, die sich bald vom Arbeitsmarkt zurückziehen, gemeinsam einen Arbeitsplatz mit einer/einem jungen Mitarbeiterln füllen und dabei als deren/dessen Mentor agieren. ${ }^{11}$

\section{Interimsmanagement}

\subsection{Allgemeine Charakteristika}

Beim Interimsmanagement stellt ein Arbeitgeber eine/n Arbeitnehmerln für eine befristete Zeit zur Erfüllung einer bestimmten Aufgabe an. Bei den Arbeitskräften handelt es sich um hochspezialisierte ExpertInnen, die ein bestimmtes Management- oder technisches Problem lösen oder dem Betrieb in wirtschaftlich schwierigen Zeiten helfen sollen. ${ }^{12}$ InterimsmanagerInnen übernehmen somit ähnliche Aufgaben wie externe UnternehmensberaterInnen, werden aber vom Arbeitgeber angestellt, in die Unternehmensorganisation eingebunden und haben dadurch gewisse Weisungsbefugnisse gegenüber der Kernbelegschaft, allerdings nicht im selben Ausmaß wie andere ManagerInnen im Betrieb.

Interimsmanagement ist eine in Europa kaum verbreitete Beschäftigungsform und scheint wenn, dann eher in der Privatwirtschaft Anwendung zu finden, insbesondere in Zusammenhang mit Unternehmensrestrukturierungen. In diesem Fällen suchen die Betriebe nach zusätzlichen Managementkapazitäten, da zur Bewältigung der Restrukturierung nicht genügend Ressourcen zur Verfügung stehen und/oder die benötigten Qualifikationen im Unternehmen fehlen. Interimsmanagement bietet Betrieben somit Zugang zu Spezialistenwissen und neuen Ideen, die passgenau auf die Anforderungen des Unternehmens ausgerichtet werden können, ohne gleichzeitig eine langfristige Bindung einzugehen und sich mit arbeitsrechtlichen Aspekten der Beendigung des Dienstverhältnisses auseinandersetzen zu müssen. ${ }^{13}$

Ein anderer Anwendungsbereich von Interimsmanagement ist das Füllen von Lücken, wenn das ursprüngliche Management z. B. durch Krankheit kurzfristig ausfällt oder im Fall von Pensionierungen nicht rechtzeitig nachbesetzt werden kann.

InterimsmanagerInnen sind im Allgemeinen zwischen 40 und 60 Jahre alt, ${ }^{14}$ da für die Erfüllung der ihnen übertragenen Aufgaben ein vergleichsweise hohes Maß an Fähigkeiten und Erfahrungen nötig ist. Hinsichtlich deren Motivation, ein derartiges Beschäftigungsverhältnis einzugehen, kann keine eindeutige Aussage getroffen werden. Manche ExpertInnen gehen davon aus, das InterimsmanagerInnen bewusst diese Art von Arbeit wählen, z. B. um eine bessere Work-Life-Balance zu erzielen oder 
weil sie die Abwechslung und Herausforderung, immer wieder in neuen Unternehmen tätig zu sein, bevorzugen. ${ }^{15}$ Andere gehen hingegen davon aus, dass InterimsmanagerInnen vielfach keine andere Wahl haben, z. B. da sie ihren letzten Arbeitsplatz verloren haben und keine andere, stabilere Beschäftigung finden können. ${ }^{16}$

\subsection{Auswirkungen auf die Arbeitsbedingungen und den Arbeitsmarkt}

Auf Grund der Tatsache, dass Interimsmanagement durch ein befristetes Arbeitsverhältnis charakterisiert ist, ergibt sich eine geringere Beschäftigungsstabilität als in Standardbeschäftigungsverhältnissen. Allerdings zeigt sich, dass dies im Allgemeinen durch ein höheres Einkommen im Vergleich zu anderen ManagerInnen kompensiert wird. ${ }^{17}$ Des Weiteren profitieren InterimsmanagerInnen vielfach von einem hohen Maß an Autonomie und Flexibilität, auch hinsichtlich Arbeitszeit und -ort.

Von InterimsmanagerInnen wird erwartet, dass sie sich schnell und effizient an die neue Unternehmenskultur und die innen übertragene Aufgabe anpassen und die Kernbelegschaft unterweisen, obwohl sie selbst nur eingeschränkt in das Unternehmen integriert sind. Das kann zu psychosozialem Stress führen. ${ }^{18}$ Darüber hinaus haben sie in vielen Fällen keinen bzw. eingeschränkten Zugang zu Weiterbildungsmaßnahmen, die vom Unternehmen organisiert bzw. finanziert werden. ${ }^{19}$

Das hohe Qualifikationsniveau und die Expertise der InterimsmangerInnen haben vielfach wissensgenerierende Effekte innerhalb der Kernbelegschaft der betroffenen Unternehmen. Die Anwesenheit der InterimsmanagerInnen fördert Dynamik und Innovation im Betrieb und beeinflusst unter Umständen sogar die Unternehmenskultur. Im Allgemeinen wird davon ausgegangen, dass Interimsmanagement die Wettbewerbsfähigkeit und Nachhaltigkeit der Unternehmen positiv beeinflusst, was - langfristig betrachtet - zu Unternehmenswachstum und Arbeitsplatzwachstum führen kann.

\section{Gelegenheitsarbeit}

\subsection{Allgemeine Charakteristika}

Gelegenheitsarbeit beschreibt eine Beschäftigungsform, in der der Arbeitgeber nicht verpflichtet ist, die unselbstständig beschäftigten Arbeitnehmerlnnen kontinuierlich bzw. regelmäßig mit Arbeiten zu beauftragen, sondern sie bei Bedarf einberufen kann. ${ }^{20}$ Das Unternehmen rekrutiert einen Pool an MitarbeiterInnen, die, falls kurzfristig Personalbedarf entsteht, kontaktiert werden. Die GelegenheitsarbeiterInnen haben dann die 
Wahl, den Arbeitsauftrag anzunehmen oder abzulehnen. In letzterem Fall werden so lange weitere Kandidaten kontaktiert, bis ein/e verfügbare Mitarbeiterln gefunden ist.

Eurofound (2015) unterscheidet zwei Arten von Gelegenheitsarbeit, die in den Mitgliedsstaaten, in denen sie anzutreffen sind, weitgehend durch gesetzliche oder kollektivvertragliche Regelungen determiniert sind:

- Sporadische Beschäftigungsverhältnisse, bei denen ein Arbeitgeber regelmäßig oder unregelmäßig an Arbeitnehmerlnnen herantritt, um eine bestimmte Aufgabe zu erfüllen. Es handelt sich um eine spezielle Art von befristeten Arbeitsverhältnissen, die teilweise durch sehr kurze Dauer gekennzeichnet sind. Die meisten identifizierten nationalen Regelungen bestimmen Obergrenzen für die Anzahl an Tagen pro Jahr, die die Arbeitnehmerlnnen in dieser Beschäftigungsform tätig sein dürfen bzw. für die ein Arbeitgeber MitarbeiterInnen in dieser Form beschäftigen darf. In mehreren Modellen ist die Einsatzmöglichkeit auf bestimmte Branchen bzw. Tätigkeiten (insbesondere z. B. Landwirtschaft, Tourismus oder andere saisonale Aktivitäten) beschränkt.

- Arbeit auf Abruf, bei der zwar ein kontinuierliches und vielfach unbefristetes Arbeitsverhältnis besteht, die Arbeitnehmerlnnen aber nur dann zur Arbeit kommen, wenn sie vom Arbeitgeber - entsprechend dessen Bedarf und meistens kurzfristig - dazu aufgefordert werden. Manche Modelle garantieren den MitarbeiterInnen eine Mindeststundenanzahl, während bei anderen der Arbeitgeber überhaupt nicht verpflichtet ist, die Arbeitnehmerlnnen mit Arbeit zu versorgen (sogenannte Zero-Hours-Verträge). In den meisten identifizierten Modellen haben die Arbeitnehmerlnnen keinen Anspruch auf ein Mindesteinkommen bzw. Vergeltung von Wartezeiten, d. h. werden ausschließlich für die geleisteten Arbeitsstunden entlohnt.

Gelegenheitsarbeit findet sich in erster Linie in Branchen mit saisonalen Schwankungen (z. B. Landwirtschaft, Tourismus, Einzelhandel) oder in Bereichen mit anderweitig fluktuierender Auslastung (z. B. Gesundheitswesen, Pflegebereiche). In meisten Fällen findet sie für niedrig qualifizierte Tätigkeiten Anwendung und wird von jungen Arbeitnehmerlnnen, vielfach Frauen, ausgeführt.

Die Hauptmotivation für Arbeitgeber, auf Gelegenheitsarbeit zurückzugreifen, ist die Flexibilität, die diese Beschäftigungsform zur Deckung von kurzfristig auftretendem Personalbedarf bietet. ${ }^{21}$ Für einen Teil der Gelegenheitsarbeiterlnnen ist diese Art der Tätigkeit vorteilhaft, da sie innen die flexible Möglichkeit bietet, Arbeit mit anderen Aktivitäten (z. B. Betreuungspflichten, Ausbildung) zu verbinden, zusätzliches Einkommen zu generieren oder erste Erfahrungen am Arbeitsmarkt zu sammeln. ${ }^{22}$ Die meisten Gelegenheitsarbeiterlnnen dürften jedoch in diese Beschäfti- 
gungsform gedrängt werden, da keine stabileren Alternativen am Arbeitsmarkt für sie verfügbar sind.

\subsection{Auswirkungen auf die Arbeitsbedingungen und den Arbeitsmarkt}

Im Allgemeinen resultiert Gelegenheitsarbeit in einem hohen Maß an Flexibilität für den Arbeitgeber und in einem geringen Maß an Sicherheit für die Arbeitnehmerlnnen, die mit unregelmäßigen und für sie kaum planbaren Arbeitszeiten, niedrigem Einkommen und geringer sozialer Absicherung konfrontiert sind. ${ }^{23}$ Vielfach verrichten GelegenheitsarbeiterInnen repetitive Standardaufgaben mit wenig Autonomie, haben wenig Zugang zu Weiterbildungsangeboten des Arbeitgebers und erleben ihre Tätigkeit als wenig zufriedenstellend. ${ }^{24}$ Während viele Gelegenheitsarbeiterlnnen mit einem geringen Stundenausmaß (und somit auch Einkommen) konfrontiert sind, haben andere - zumindest sporadisch - eine sehr hohe Arbeitsauslastung, da sie aufgrund der Unvorhersagbarkeit des nächsten Arbeitsauftrags jede innen gebotene Arbeit annehmen.

Aus makroökonomischer Sicht kann Gelegenheitsarbeit zur Arbeitsmarktintegration bestimmter Gruppen (insbesondere von jungen ArbeitnehmerInnen, die so erste Erfahrungen sammeln können) und zur Reduktion von Schwarzarbeit beitragen. ${ }^{25}$ Dem gegenüber steht die Befürchtung, dass diese Beschäftigungsform Standardarbeitsverhältnisse ersetzt und dadurch langfristig zu einer Abwärtsspirale hinsichtlich der Arbeitsbedingungen führen kann. ${ }^{26}$ Des Weiteren besteht das Potenzial, das Gelegenheitsarbeit die Segmentierung am Arbeitsmarkt vorantreibt, da in erster Linie bestimmte Arbeitnehmerlnnengruppen mit dieser im Allgemeinen prekären Beschäftigungsform konfrontiert sind.

\section{IKT-gestützte, mobile Tätigkeiten}

\subsection{Allgemeine Charakteristika}

Aufgrund der steigenden Verbreitung von modernen Informations- und Kommunikationstechnologien (IKT) und Internetzugang sowie neuen Formen der Arbeitsorganisation zeigt sich in den letzten Jahren ein höheres Ausmaß an „virtuellen, unsichtbaren ArbeitnehmerInnen, die überall digital arbeiten“. ${ }^{27}$ Im Folgenden werden unter „IKT-gestützten, mobilen Tätigkeiten" jene verstanden, die von unselbstständig Beschäftigten oder Selbstständigen zumindest teilweise, aber regelmäßig, außerhalb der Räumlichkeiten des Arbeitgebers, Kunden oder Heimarbeitsplatzes durchgeführt werden (somit mobiler als die klassische Telearbeit). Dabei werden IKT verwendet, um extern auf ein gemeinsames Datennetzwerk 
zuzugreifen. ${ }^{28}$ Die Arbeit wird an jenem Ort und zu jenem Zeitpunkt erledigt, die den Arbeitnehmerlnnen, Aufgabeninhalten und -erfordernissen am besten entsprechen.

Aufgrund einer fehlenden spezifischen rechtlichen Grundlage werden IKT-gestützte, mobile Tätigkeiten auf Basis von Standardverträgen durchgeführt und informell zwischen Arbeit- (oder Auftrag-)geber und Arbeitnehmerln vereinbart und implementiert. Um diese Beschäftigungsform anwenden zu können, müssen folgende Voraussetzungen erfüllt sein:

- Die Aufgaben müssen ortsunabhängig erledigt werden können.

- Technische Infrastruktur, die es den Arbeitnehmerlnnen ermöglicht, auf benötigte Informationen zuzugreifen und sich mit KollegInnen und Kunden auszutauschen, muss verfügbar sein.

- Die Unternehmenskultur muss durch ein gewisses Maß an gegenseitigem Vertrauen zwischen Vorgesetzten und Untergebenen gekennzeichnet sein.

- Die betroffenen Arbeitnehmerlnnen müssen ein gewisses Maß an Selbstorganisationsfähigkeiten aufweisen und gewillt sein, ihre Arbeit unabhängig zu erledigen.

Bei den mobilen Mitarbeiterlnnen handelt es sich in erster Linie um höher qualifizierte Männer im mittleren Alterssegment, oftmals in Managementpositionen im Dienstleistungssektor. ${ }^{29}$ In vielen Fällen werden derartige Beschäftigungsverhältnisse auf Wunsch der MitarbeiterInnen etabliert, die dadurch mehr Flexibilität und eine bessere Balance zwischen Berufs- und Privatleben erreichen möchten. Die Arbeitgeber stimmen zu, um die Arbeitskräfte nicht an die Konkurrenz zu verlieren und um innovative Arbeitspraktiken umzusetzen, die zu Effizienz- und Produktivitätsgewinnen führen könnten.

\subsection{Auswirkungen auf die Arbeitsbedingungen und den Arbeitsmarkt}

IKT-gestützte, mobile Tätigkeiten bringen ein hohes Maß an Autonomie und Flexibilität (vor allem in Bezug auf Arbeitszeit und -ort) für die ArbeitnehmerInnen mit sich ${ }^{30}$ und können zu einer persönlichen Effizienz- und Produktivitätsverbesserung führen, da die Mitarbeiterlnnen selbst entscheiden, wann sie welche Aktivitäten wo durchführen.

Gleichzeitig kann es zu einer Arbeitsintensivierung kommen, insbesondere, wenn die Entlohnung aufgrund des dieser Beschäftigungsform inhärenten Kontrollverlusts des Arbeitgebers auf Basis des Arbeitsergebnisses anstatt der Arbeitszeit beruht. Wird die Arbeit oft von zu Hause aus erledigt, besteht außerdem das Potenzial eines Verschwimmens der Grenzen von Arbeit und Privatleben.

Trotz des starken Einsatzes von IKT können mobile Arbeitnehmerlnnen 
eine persönliche und professionelle Isolation erleben, da die physische Interaktion mit Vorgesetzten, Untergebenen, KollegInnen oder Kunden eingeschränkt ist und/oder sie nicht (mehr) in den gesamten Organisationsprozess eingebunden sind.

Je nachdem, wie die bilaterale Vereinbarung zu IKT-gestützten, mobilen Tätigkeiten zwischen Arbeitgeber und Arbeitnehmerlnnen ausgestaltet ist (bzw. in Abhängigkeit von der Unternehmenskultur), kann es vorkommen, dass von mobilen MitarbeiterInnen eine 24-Stunden-Erreichbarkeit erwartet wird bzw. die ArbeitnehmerInnen eine derart umfassende Erreichbarkeit vermuten und dementsprechend handeln. ${ }^{31}$ Sollte dies der Fall sein, sind negative Auswirkungen auf die Work-Life-Balance und die psychische und physische Gesundheit der Arbeitnehmerlnnen zu erwarten.

Des Weiteren ist zu erwähnen, dass wegen der physischen Absenz der Mitarbeiterlnnen in den Räumlichkeiten des Arbeitgebers klassische Arbeitgeberverantwortlichkeiten hinsichtlich Maßnahmen zur Erhaltung der Gesundheit und Sicherheit an die Arbeitnehmerlnnen ausgelagert werden. Ein Arbeitgeber kann beispielsweise lediglich Empfehlungen in Bezug auf die ergonomische Gestaltung des Heimarbeitsplatzes geben, die Implementierung kann jedoch ausschließlich durch die Mitarbeiterlnnen erfolgen.

Aus makroökonomischer Sicht kann durch den fortschreitenden Einsatz von IKT davon ausgegangen werden, dass IKT-gestützte, mobile Tätigkeiten in Zukunft weitere Verbreitung finden werden. In Ländern, in denen diese Beschäftigungsform bereits besser etabliert ist, wurde beobachtet, dass sie die Arbeitswelt „revolutioniert" hat. ${ }^{32}$ Es kam bzw. kommt zur Entwicklung neuer Arbeitsprozesse und -organisationen, zu einer sinkenden Bedeutung der physischen Anwesenheit an einem bestimmten Arbeitsplatz und zur Kompetenzentwicklung der Mitarbeiterlnnen, insbesondere hinsichtlich transversaler Fähigkeiten. Darüber hinaus kann diese Beschäftigungsform zur Arbeitsmarktintegration benachteiligter Gruppen beitragen, z. B. jener Personen, die aufgrund von gesundheitlichen oder persönlichen Gründen nicht zu bestimmten Kernarbeitszeiten arbeiten können oder wollen, oder jener, die wegen der geografischen Lage ihres Wohnsitzes nur bedingt Zugang zu Arbeitsplätzen haben. ${ }^{33}$

Gleichzeitig muss jedoch berücksichtigt werden, dass derartige Tätigkeiten nicht von allen Arbeitnehmerlnnen durchgeführt werden können, und insbesondere jene, die sich nicht an die technologischen Entwicklungen anpassen können, vom Arbeitsmarkt ausgeschlossen werden könnten. 


\section{Arbeit auf Basis von Gutscheinsystemen}

\subsection{Allgemeine Charakteristika}

Bei dieser Beschäftigungsform (in Österreich: Dienstleistungsscheck) erwirbt ein Arbeitgeber einen Gutschein von einer dritten Partei (im Allgemeinen einer öffentlichen Einrichtung), der für die Bezahlung der Dienstleistungen des/der Arbeitnehmerln verwendet wird. Die Beschäftigten können den Gutschein anschließend z. B. in einer Bank oder Postfiliale einlösen und erhalten ihr Nettoentgelt ausbezahlt. Die entsprechenden Steuern und Sozialversicherungsbeträge werden von der auszahlenden Stelle an die relevanten Behörden abgeführt.

Die derartigen in Europa identifizierten Modelle finden in erster Linie in der Landwirtschaft und für Dienstleistungen in privaten Haushalten Anwendung, da in diesen Bereichen ein großer Teil der Schwarzarbeit stattfindet und die Regierungen diese Systeme als Instrument zur Bekämpfung von Schwarzarbeit eingeführt haben. Die Überlegung dahinter ist, Arbeitgebern eine unbürokratische und leicht zu administrierende Möglichkeit zu geben, Beschäftigte offiziell anzustellen, ohne formale Arbeitsverträge etablieren zu müssen.

In den meisten europäischen Ländern, in denen Arbeit auf Basis von Gutscheinsystemen organisiert werden kann, ist eine Obergrenze der Anwendbarkeit (z. B. Anzahl der Gutscheine, die ein Arbeitgeber erwerben kann, Anzahl der Arbeitstage pro Arbeitnehmerln) vorgesehen, um zu vermeiden, dass durch diese flexible Beschäftigungsform andere Vertragsarten verdrängt werden. In ähnlicher Weise etablieren die meisten Modelle einen Mindeststundenlohn sowie zumindest eine Teilversicherung in der Sozialversicherung, um die Ausbeutung der Arbeitnehmerlnnen zu vermeiden.

Bei der Mehrheit der ArbeitnehmerInnen auf Basis von Gutscheinsystemen handelt es sich um Frauen. Es zeigt sich eine Tendenz zu entweder jüngeren (unter 40) oder älteren (über 50) Arbeitnehmerlnnen, meistens im niedrig qualifizierten Segment. Die verfügbaren Daten deuten darauf hin, dass die auf Basis von Gutscheinsystem Beschäftigten diese Art von Erwerbstätigkeit neben einem anderen Beschäftigungsstatus (z. B. unselbstständig beschäftigt, arbeitslos, pensioniert) realisieren.

\subsection{Auswirkungen auf die Arbeitsbedingungen und den Arbeitsmarkt}

Der größte Vorteil von Arbeit auf Basis von Gutscheinsystemen ist die Legalisierung von Tätigkeiten, die oft in Form von Schwarzarbeit durchgeführt werden, da durch die einfache Handhabung ein Anreiz für die Arbeitgeber gesetzt wird, ein formales Beschäftigungsverhältnis anzubieten. 
Wenngleich die verfügbaren Daten zeigen, dass derartige Modelle durchaus einen Beitrag zur Bekämpfung von Schwarzarbeit und der Arbeitsmarktintegration benachteiligter Gruppen (insbesondere von niedrig Qualifizierten, jungen ArbeitnehmerInnen, Arbeitslosen und Personen mit Migrationshintergrund) leisten, kann damit - zumindest bislang - nicht dem gesamten Problem umfassend entgegengewirkt werden.

Die Arbeitnehmerlnnen erhalten Zugang zur Sozialversicherung und vielfach auch die Gewährleistung eines Mindestlohns. Im Allgemeinen haben die Beschäftigten die Möglichkeit, ihre Tätigkeiten weitgehend autonom auszuführen, zu wählen, welche Aufträge sie annehmen oder nicht, und profitieren so häufig von kurzen Arbeitswegen und Arbeitszeiten, die sich gut mit anderen Verpflichtungen (wie z. B. Kinderbetreuung oder Studium) vereinbaren lassen.

Demgegenüber steht, dass derartige Beschäftigungsverhältnisse als Gelegenheitsarbeit (siehe oben) klassifiziert werden können, somit durch ein hohes Maß an Beschäftigungs- und Einkommensunsicherheit für die Arbeitnehmerlnnen gekennzeichnet sind. Hinzu kommt, dass insbesondere bei Tätigkeiten in privaten Haushalten adäquate Arbeitsbedingungen nicht immer und Weiterbildungsmaßnahmen sowie die Möglichkeit zum Austausch mit KollegInnen nahezu nie gegeben sind.

\section{Portfolioarbeit}

\subsection{Allgemeine Charakteristika}

Portfolioarbeit beschreibt die Tätigkeit von Selbstständigen, die eine Vielzahl an kleinen Aufträgen für zahlreiche Kunden erledigen. Clinton et al. (2006) fassen die folgenden Aspekte als Hauptmerkmale zusammen:

- Es handelt sich um selbstorganisierte, unabhängige, einkommengenerierende Arbeit, die die Fähigkeit zur Selbstvermarktung voraussetzt.

- Portfolioarbeit beinhaltet den Aufbau und die Pflege einer Vielzahl an Kundenbeziehungen und die Notwendigkeit, sich kontinuierlich an unterschiedliche Arbeitssituationen und Kundenwünsche anzupassen.

- Das Spektrum an Tätigkeiten findet nicht in einer einzelnen Organisation statt.

Portfolioarbeit dürfte insbesondere durch die steigende Anzahl an (freiwilligen und unfreiwilligen) Selbstständigen seit der Wirtschaftskrise sowie durch den beobachtbaren Trend zu hoch qualifizierten Spezialistlnnen, die ihre Tätigkeit als Freelancer anbieten, um höhere Flexibilität und Einkommen erzielen zu können, motiviert sein. ${ }^{34}$

Portfolioarbeit findet sich insbesondere in der Kreativwirtschaft sowie in professionellen, technischen und wissenschaftlichen Berufen, dem Realitätenwesen und dem Informations- und Kommunikationssektor. ${ }^{35}$ Es lässt 
sich eine gewisse Tendenz zu männlichen Arbeitnehmerlnnen im jüngeren bis mittleren Alterssegment beobachten, die diese Form der Tätigkeit bewusst wählen, um sich selbst mit interessanten und herausfordernden Arbeiten zu versorgen.

\subsection{Auswirkungen auf die Arbeitsbedingungen und den Arbeitsmarkt}

Da es sich bei Portfolioarbeit um eine spezielle Ausgestaltung der Selbstständigkeit handelt, ist sie durch ein hohes Maß an Autonomie und Flexibilität gekennzeichnet, gleichzeitig aber auch durch Beschäftigungsund Einkommensunsicherheit und vielfach durch geringeren sozialen Schutz im Vergleich zu unselbstständig Beschäftigen.

Portfolioarbeiterlnnen scheinen diese Art der Tätigkeit zu genießen, erleben ihre Arbeit als bedeutend und bewerten ihre Arbeitszufriedenheit hoch, da sie die Möglichkeit haben, neue Herausforderungen zu bewältigen, ihre verschiedenen Fähigkeit so gut wie möglich einzusetzen und kontinuierlich neue Kenntnisse zu erlangen.

Es gibt aber auch Fälle, in denen die Arbeitsaufträge nicht den Fähigkeiten und Erfahrungen entsprechen, und die nur angenommen werden, um Einkommen zu generieren. ${ }^{36}$ Diese sind naturgemäßig weniger zufriedenstellend und können sogar als sozialer Abstieg erlebt werden.

Britische Studien deuten darauf hin, dass Portfolioarbeit das Potenzial hat, als Instrument zur Einkommensgenerierung nach der Pensionierung zu dienen, da es den Arbeitnehmerlnnen die Flexibilität bietet, selbst zu wählen, welche Tätigkeiten angenommen werden und welche nicht. ${ }^{37}$ Dies kann der psychischen Gesundheit der Beschäftigten zuträglich sein, da sie auch nach der Pensionierung die Möglichkeit haben, ihre angeeigneten Fähigkeiten, Fertigkeiten und Erfahrungen anzuwenden und sich so weiterhin als wertvolles Mitglied der Gesellschaft zu fühlen.

\section{Crowdsourcing}

\subsection{Allgemeine Charakteristika}

Beim Crowdsourcing nutzen Organisationen und Individuen OnlinePlattformen, um an andere Organisationen oder Individuen zur Lösung eines bestimmten Problems oder Bereitstellung einer bestimmten Dienstleistung gegen Entgelt heranzutreten. ${ }^{38}$ Es handelt sich somit um eine neue Art von Auslagerung, bei der einzelne, in den meisten Fällen sehr kleine, Aufgaben an ArbeitnehmerInnen ausgelagert und über das Internet erledigt werden. ${ }^{39}$ Beispiele für derartige Aufgaben sind die Entwicklung von Webinhalten und -software, der Aufbau und die Pflege von Da- 
tenbanken, die Kategorisierung von Webseiten oder Bildern und die Suche nach bestimmten Kriterien, Transkriptionen, Übersetzungen, Korrekturlesen von Dokumenten oder das Design von Logos, Werbesprüchen und Broschüren. ${ }^{40}$

Bei den meisten Crowdsourcing-Plattformen initiiert der Auftraggeber das Beschäftigungsverhältnis, indem er eine Anfrage zur Erbringung der gewünschten Tätigkeit auf der Plattform publiziert. Dies kann in Form eines Wettbewerbs (bei dem die Arbeitnehmerlnnen unmittelbar die gewünschte Dienstleistung erbringen und der Auftraggeber die für inn beste Lösung wählt und ausschließlich diese entlohnt) oder ähnlich einer Ausschreibung (bei der die Arbeitnehmerlnnen einen Vorschlag unterbreiten, wie und unter welchen Konditionen sie planen, die gewünschte Leistung zu erbringen, und der Auftraggeber auf Basis dieser Beschreibungen einen konkreten Auftrag erteilt) erfolgen. Es gibt jedoch auch Plattformen, auf denen Arbeitnehmerlnnen ihre Dienste anbieten und Auftraggeber im Anlassfall unter den Angeboten das für sie passendste wählen.

Die Plattform agiert als Intermediär, der Angebot und Nachfrage zusammenbringt, aber nicht direkt in die Geschäftsverbindung zwischen Auftraggeber und Auftragnehmer eingebunden ist. Diese beruht auf einer individuellen Vereinbarung zwischen den beiden Parteien (nicht unbedingt ein formaler Vertrag), wobei einige Plattformen unverbindliche oder verbindliche Vorgaben bezüglich Preis, Lieferzeit, geistiger Eigentumsrechte oder anderer relevanter Aspekte anführen.

Abbildung 3: Arbeitsmethode von virtuellen Plattformen für Crowdsourcing

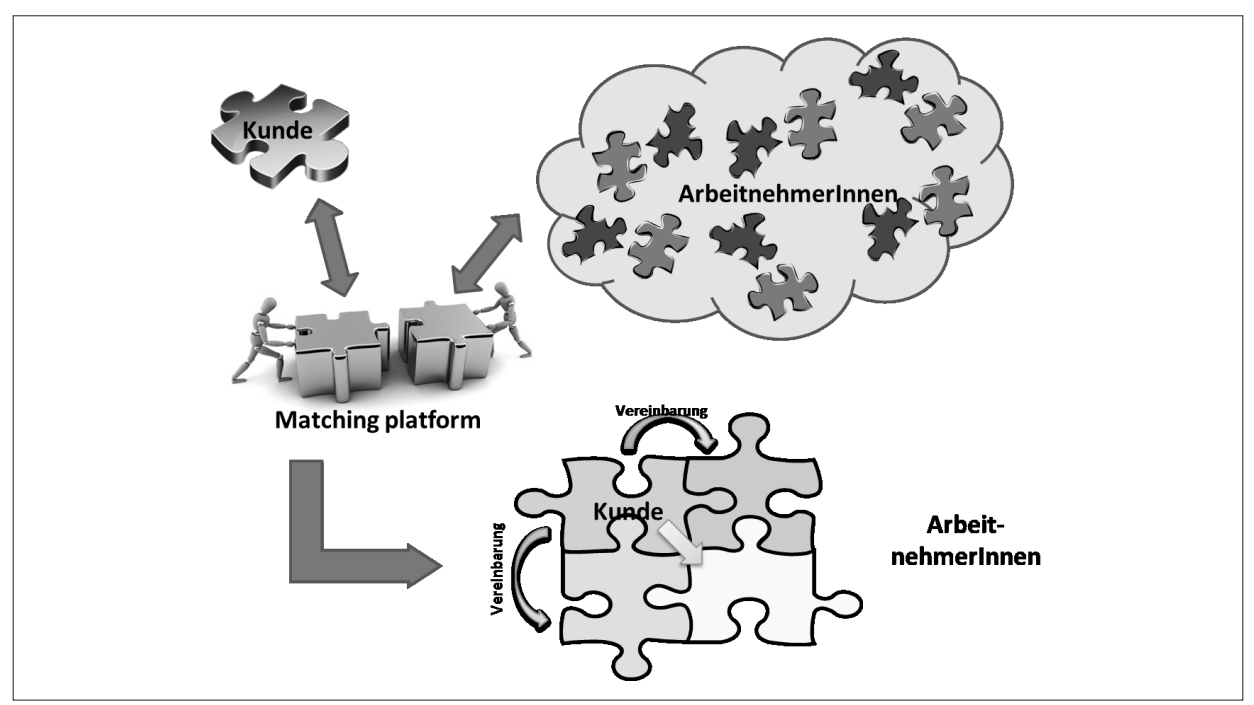

Quelle: Eurofound (2015). 
Die Beschäftigten, die Aufträge über Crowdsourcing-Plattformen annehmen, sind eher jung (bis ca. Mitte 30). Jene ArbeitnehmerInnen, die im Rahmen der Eurofound-Studie (2015) befragt wurden, führen diese Tätigkeiten neben anderen (unselbstständige Beschäftigung, Studium, Kinderbetreuungspflichten) aus, um auf flexible Weise zusätzliches Einkommen zu generieren, weil innen diese Arbeit Spaß macht und weil sie sich davon Lerneffekte und professionelle Weiterentwicklung erhoffen. ${ }^{41}$

Die Auftraggeber nutzen diese Beschäftigungsform in erster Linie, um aus einem breiten Pool potenzieller Auftragnehmer jene zu wählen, die die für sie passendsten Dienste anbieten. Dabei spielen vor allem die Kriterien der verfügbaren Qualifikation/Expertise, des angebotenen Preises, der Dauer der Leistungserbringung sowie der Aspekt, dass keine arbeitsrechtliche Beziehung eingegangen werden muss, eine Rolle. ${ }^{42}$

\subsection{Auswirkungen auf die Arbeitsbedingungen und den Arbeitsmarkt}

Im Allgemeinen kann davon ausgegangen werden, dass die Arbeitsbedingungen von Beschäftigten, die Aufträge über Crowdsourcing-Plattformen annehmen, nicht sehr gut sind, und das Potenzial besteht, dass stabilere Beschäftigungsverhältnisse (zumindest in Bezug auf bestimmte Berufe oder einzelne Tätigkeiten innerhalb von Berufen) langfristig durch diese prekärere Beschäftigungsform ersetzt werden.

Crowdsourcing kann als Form der Gelegenheitsarbeit (siehe oben) betrachtet werden, da die Arbeitnehmerlnnen mit einem hohen Maß an Beschäftigungs- und somit Einkommensunsicherheit konfrontiert sind. Hinzu kommt, dass die Entlohnung vielfach sehr gering und im Fall von Wettbewerben auch nicht garantiert ist. ${ }^{43}$ Eine Studie über die CrowdsourcingPlattform „Amazon Mechanical Turk“ hat beispielsweise ergeben, dass $90 \%$ der beauftragten Tätigkeiten für weniger als $\$ 0,10$ ( $€ 0,07$ ) erbracht werden. ${ }^{44}$ Derart niedrige Sätze sind einerseits auf die Tatsache zurückzuführen, dass die einzelnen Aufgaben oft sehr klein sind. Andererseits ist die Konkurrenz um die Aufträge teilweise so hoch, dass die Auftraggeber keine höheren Preise akzeptieren müssen.

Dieser Umstand wird dadurch verschärft, dass bei den CrowdsourcingPlattformen eine gewisse Informationsasymetrie vorherrscht, $d$. h. den Beschäftigten im Vorfeld nicht unbedingt das volle Ausmaß an Informationen vorliegt, das für eine solide Einschätzung des zu erwartenden Arbeitsausmaßes nötig ist. ${ }^{45}$ Somit kann es vorkommen, dass deutlich mehr Zeit investiert werden muss, wodurch die (relative) Entlohnung geringer ist als erwartet.

Da die Beschäftigten als Selbstständige betrachtet werden, liegt es in ihrer eigenen Verantwortung, für soziale Absicherung, Weiterbildungs- 
maßnahmen und ähnliches zu sorgen und ihr Einkommen korrekt zu versteuern.

Des Weiteren ist nicht immer gewährleistet, dass das geistige Eigentum der Arbeitnehmerlnnen geschützt ist bzw. sie Zugang zu Konfliktlösungsmechanismen haben, falls Probleme mit den Kunden auftreten.

Crowdsourcing hat jedoch auch positive Elemente. Da es den Beschäftigten frei steht, jene Aufträge zu wählen, die für sie am geeignetsten sind, wählen sie im Allgemeinen jene, mit denen sie Effizienz- und Produktivitätsgewinne realisieren können. Aufgrund der „virtuellen Leistungserbringung" erleben sie ein hohes Maß an Autonomie und Flexibilität, wodurch im Idealfall auch eine gute Balance zwischen Berufs- und Privatleben erzielt werden kann.

Crowdsourcing bietet ArbeitnehmerInnen die Möglichkeit, zusätzliches Einkommen zu generieren und kann insbesondere jungen Beschäftigten zu einer besseren Integration in den Arbeitsmarkt verhelfen, da sie durch diese Art von Beschäftigung die von vielen Arbeitgebern geforderten Erfahrungen sammeln und Lerneffekte realisieren können. In ähnlicher Weise wurde beobachtet, dass Crowdsourcing in Gegenden mit stagnierender Wirtschaftsentwicklung den Zugang zu Beschäftigung und Einkommensgenerierung erleichtert, da durch diese Beschäftigungsform Arbeit unabhängig vom geografischen Standort angenommen werden kann. ${ }^{46}$

\section{Kollaboratives Arbeiten}

\subsection{Allgemeine Charakteristika}

Kooperation und Zusammenarbeit ist eine traditionelle Arbeitform unter Selbstständigen und KMU, um die Einschränkungen, die mit der geringeren Größe verbunden sind, zu überwinden. Die Eurofound-Studie (2015) identifiziert drei Arten von Kooperationen, die kürzlich verstärkt zu beobachten sind und über eine klassische Geschäftspartner-Beziehung oder Kooperationen entlang der Wertschöpfungskette hinausgehen:

- Schirmgesellschaften bieten Selbstständigen oder KMU bestimmte administrative Dienstleistungen, wie z. B. Rechnungslegung oder Versteuerung, während die maßgeblichen Kennzeichen der Selbstständigkeit (Unabhängigkeit, Risiko, Autonomie, Kontrolle etc.) erhalten bleiben. In einigen europäischen Modellen ermöglicht die Teilnahme an einer Schirmgesellschaft den Selbstständigen Zugang zu einem vorteilhafteren Sozialversicherungsregime (vielfach eine Position zwischen unselbstständig Beschäftigten und „Standard-Selbstständigen"). Die wenigen verfügbaren Daten deuten darauf hin, dass insbesondere Selbstständige im mittleren bis älteren Alterssegment, die in der Kreativwirtschaft oder in professionellen, technischen und wissen- 
schaftlichen Bereichen tätig sind, an Schirmgesellschaften teilnehmen.

- Beim Coworking teilen sich Selbstständige oder Mikrounternehmen Räumlichkeiten und unterstützende Leistungen wie etwa Rezeption oder Sekretariat. Die Mietkonditionen sind vielfach sehr flexibel, sodass die Selbstständigen nicht nur ein für sie passendes „Paket“ indviduell zusammenstellen, sondern dieses auch kontinuierlich entsprechend ihres Bedarfs adaptieren können. Es handelt sich dabei aber nicht ausschließlich um ein „Unternehmenshotel“, sondern es wird intensivere Kooperation unter den Beteiligten sowie sozialer und professioneller Austausch angeregt. Dies erfolgt z. B. durch die Bereitstellung von Besprechungszimmern oder Treffplätzen wie Cafés und Kantinen oder die Organisation von gemeinsamen Schulungen, Seminaren oder Diskussionsrunden. Wenngleich die meisten Coworking-Zentren keine Einschränkungen bezüglicher ihrer Mitglieder treffen, zeigt sich, dass insbesondere Selbstständige in der Kreativwirtschaft oder in beratenden Berufen auf diese Beschäftigungsform zurückgreifen. Die Coworker sind im Allgemeinen hoch qualifiziert und im jüngeren bis mittleren Alterssegment.

- Genossenschaften sind keine „neuen Beschäftigungsformen“, wie in der Eurofound-Studie (2015) verstanden, wurden jedoch in die Analysen am Rande aufgenommen, da es vereinzelt zur Entwicklung neuer Genossenschaftsformen, inklusive Genossenschaften für Selbstständige, kommt.

\subsection{Auswirkungen auf die Arbeitsbedingungen und den Arbeitsmarkt}

Die analysierten Formen des kollaborativen Arbeitens von Selbstständigen ändert nichts an deren Status als Selbstständige, wodurch sich keine gravierenden Auswirkungen auf ihre Arbeitsbedingungen und ihre soziale Absicherung ergeben. Ein wesentlicher Vorteil, den jedoch alle drei Formen bieten, ist die Möglichkeit, sich mit Gleichgesinnten auszutauschen, geschäftliche Kooperationen aufzubauen und Zugang zu bestimmten Leistungen zu erlangen, die sie sonst selbst organisieren und finanzieren müssten. Durch dieses Potenzial, Synergieeffekte zu generieren und dadurch Effizienz- und Produktivitätssteigerungen zu realisieren, kann es auch zu einer Verbesserung der Work-Life-Balance der Selbstständigen kommen.

Ein wesentlicher Punkt ist dabei auch, dass das kollaborative Arbeiten dabei hilft, nicht nur die berufliche, sondern auch die persönliche Isolation, mit der viele Selbstständige (insbesondere, wenn sie von zu Hause aus arbeiten) konfrontiert sind, zu überwinden. Das höhere Ausmaß an sozialer 
Interaktion kann darüber hinaus dazu beitragen, dass persönliche und transversale Kompetenzen („soft skills“) weiterentwickelt werden, was sich positiv auf die Beschäftigungsfähigkeit auswirken kann.

Aus makroökonomischer Sicht besteht das Potenzial, dass durch die verstärkte Kooperation von Selbstständigen qualitativ hochwertigere Leistungen erbracht sowie Innovationen gefördert werden.

Kollaboratives Arbeiten kann auch zu einem dynamischeren Arbeitsmarkt beitragen, da der Schritt in die Selbstständigkeit dadurch in gewisser Weise erleichtert wird und gegebenenfalls eine höhere und nachhaltigere Teilnahme am Arbeitsmarkt erreicht werden kann.

\section{Abschließende Betrachtungen}

Die Ergebnisse der Eurofound-Studie weisen darauf hin, dass die europäischen Arbeitsmärkte nach wie vor von „Standardbeschäftigung" dominiert sind, sich jedoch eine steigende Heterogenität an Beschäftigungsformen beobachten lässt. Die meisten davon werden vom Wunsch oder dem Bedarf von Arbeitgebern und/oder ArbeitnehmerInnen nach mehr Flexibilität angetrieben, weshalb davon ausgegangen werden kann, dass zumin-

Abbildung 4: Zusammenfassende Darstellung der potenziellen Auswirkungen der neuen Beschäftigungsformen auf die Arbeitsbedingungen und den Arbeitsmarkt

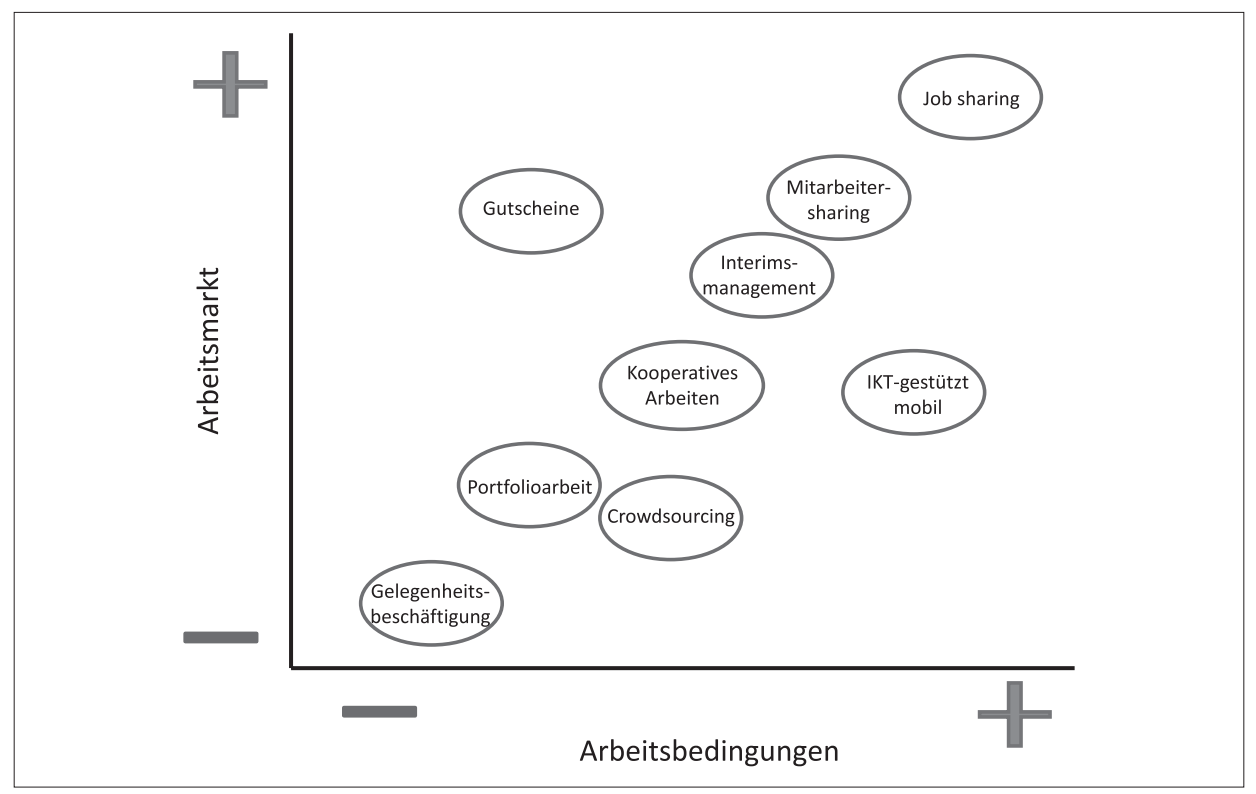

Quelle: Eurofound (2015). 
dest einige von ihnen auch in Zukunft Anwendung finden werden. Die größten Zukunftstrends sind dabei vermutlich Gelegenheitsarbeit, IKT-gestützte, mobile Tätigkeiten und Crowdsourcing.

Die diskutierten neuen Beschäftigungsformen unterscheiden sich untereinander zumindest teilweise deutlich, sowohl in Bezug auf ihre allgemeine Charakteristika und ihren Anwendungsbereich als auch hinsichtlich der potenziellen Auswirkungen auf die Arbeitsbedingungen und den Arbeitsmarkt. Während einige der identifizierten neuen Trends das Potenzial haben, Vorteile sowohl für Arbeitgeber als auch ArbeitnehmerInnen zu realisieren, dürften sich andere negativer für die Beschäftigten auswirken. Es gilt, diese potenziellen Auswirkungen genauer zu erkunden und entsprechende Handlungen zu setzen.

\section{Anmerkungen}

1 Eurofound (2015).

2 Wölfing et al. (2007).

3 Europäisches Ressourcenzentrum der Arbeitgeberzusammenschlüsse (2008); Delalande und Buannic (2006).

4 Antoine und Rorive (2006); Vötsch und Titz (2011).

5 Wölfing et al. (2007); Antoine und Rorive (2006).

6 Dubourg et al. (2006).

7 Wheatly (2013).

8 Branine (2003); Guglielmo (2008); Daniels (2011).

9 Branine (2003, 2004).

10 Wheatley (2013).

11 Ebendort.

12 Bruns und Kabst (2005; Inkson et al. (2001); Isidor et al. (2014); Russam GMS (2012).

13 Jas (2013); Inkson et al. (2001).

14 Russam GMS (2012).

15 Pollitt (2008).

16 Inkson et al. (2001).

17 Ebendort.

18 Jas (2013).

19 Inkson et al. (2001).

20 Europäisches Parlament (2000).

21 ILO (2004); Rubery et al. (2014).

22 ILO (2004).

23 ILO (2004); Pennycook et al. (2013).

24 ILO (2004); Layte et al. (2008), De Graaf-Zijl (2012); Håkansson (2001).

25 Andersson und Wadensjö (2004); Karlsson (2009).

26 Håkansson (2001); Eurofound (2008).

27 Popma (2013); Holtgrewe (2014).

28 Andriessen und Vartiainen (2006); Europäische Kommission (2010); Eurofound (2012).

29 Eurofound (2012); Schaffers et al (2006); Europäische Kommission (2010); Drobnjak und Jereb (2007).

30 Deutscher Bundestag (2013); Vinnova (2007).

31 Maschke et al. (2014); Europäische Kommission (2010); Popma (2013); Unionen (2013). 
32 Vinnova (2007; Alasoini et al. (2012).

33 Government of Norway (2011).

34 Leighton und Brown (2013).

35 Platman (2004); Deuze (2008); Gold und Fraser (2001); Leighton und Brown (2013).

36 Cohen und Mallon (1999); Gold und Fraser (2001); Platman (2004); Wild (2012).

37 Platman (2004); Wild (2012).

38 Green und Barnes (2013); Papsdorf (2009).

39 Felstiner (2011).

40 Horton und Chilton (2010); Felstiner (2011).

41 Klebe und Neugebauer (2014); Silberman et al. (2010); Ipeirotis (2011).

42 Klebe und Neugebauer (2014); Felstiner (2011).

43 Felstiner (2011); Silberman et al. (2010); Klebe und Neugebauer (2014).

44 Irani und Silberman (2013).

45 Felstiner (2011); Klebe und Neugebauer (2014).

46 Kittur et al. (2013); Felstiner (2011).

\section{Literatur}

Alasoini, T.; Järvensivu, A.; Mäkitalo, J., Suomen työelämä 2030. Miten ja miksi se on toisennäköinen kuin tällä hetkellä, Työ-ja elinkeinoministeriön raportteja, 14/2012 (Helsinki 2012).

Andersson, P.; Wadensjö, E., Hur fungerar bemanningsbranschen (How does industry staff itself?) (IFAU - Institutet för Arbetmarknadspolitisk Utvärdering, Uppsala 2004).

Andriessen, J. H.; Vartiainen, M., Mobile Virtual Work. A new paradigm? (Berlin 2006).

Antoine, M.; Rorive, B., Job pools in Belgium, Monitoring Innovative Restructuring in Europe (MIRE project, Liège 2006).

Branine, M., Part-time work and job sharing in health care: is the NHS a family-friendly employer?, in: Journal of Health Organization and Management 17/1 (2003) 53-68.

Branine, M., Job sharing and equal opportunities under the new public management in local authorities, in: International Journal of Public Sector Management 17/2 (2004) 136152.

Bruns, J.; Kabst, R., Interim-Management: A Paradox for Leadership Research?, in Management Revue 15 (2005) 512-524.

Clinton, M.; Totterdell, P.; Wood, S., A grounded theory of portfolio working experiencing the smallest of small businesses, in: International Small Business Journal 24/2 (2006) 179-203.

Cohen, L.; Mallon, M., The transition from organisational employment to portfolio working: Perceptions of boundarylessness, in: Work, Employment \& Society 13/2 (1999) 329352.

Daniels, L., Job sharing at senior level: making it work. The job share project (2011); online: http://www.thejobshareproject.com/resources.html.

De Graaf-Zijl, M., Job Satisfaction and Contingent Employment, in: De Economist 160/2 (2012) 197-218.

Delalande, F.; Buannic, L., Groupements d'employeurs, mode d'emploi (Paris 2006).

Deutscher Bundestag, Achter Zwischenbericht der Enquete-Kommission „Internet und digitale Gesellschaft", Wirtschaft, Arbeit, Green IT (Berlin 2013).

Deuze, M., The professional identity of journalists in the context of convergence culture, in: Observatorio (Obs*) Journal 2/4 (2008) 848-865.

Drobnjak, S.; Jereb, E., Telework Indicators: A Case Study of Slovenia (University of Maribor, Faculty of Organisational Sciences, Maribor/Marburg 2007). 
Dubourg, L.; Ahmling, J. A.; Bujas, L., Can job sharing work for nurse managers?, in: Australian Health Review 30/1 (2006) 17-24.

Eurofound, Use and abuse of non-standard employment contracts (Dublin 2008).

Eurofound, Fifth European Working Conditions Survey: overview report (Luxemburg 2012).

Eurofound, New forms of employment (Luxemburg 2015).

Europäisches Ressourcenzentrum der Arbeitgeberzusammenschlüsse, Antwort auf die acht gemeinsamen Grundsätze für den Flexicurity-Ansatz der Europäischen Kommission. Erklärung auf der Europäischen Zusammenkunft der Arbeitgeberzusammenschlüsse beim Ausschuss der Regionen (Brüssel 2008).

Europäische Kommission, The increasing use of portable computing and communication devices and its impact on the heath of EU workers (Luxemburg 2010).

Europäisches Parlament, Atypical work in the EU, social affairs series (= SOCl 106-EN, Luxemburg 2000).

Felstiner, A., Working the Crowd: Employment and Labor Law in the Crowdsourcing Industry, in: Berkeley Journal of Employment \& Labor Law 32/1 (2011) 143-203.

Gold, M.; Fraser, J., Portfolio workers: Autonomy and control amongst freelance translators, in: Work, employment \& society 5/4 (2011) 679-697.

Government of Norway, Kompetansearbeidsplasser - drivkraft for vekst i hele landet (= NOU 2011: 3, KRD, Oslo 2011).

Green, A.; Barnes, S.-A., CrowdEmploy Part I: Crowdsourcing for paid work. An empirical investigation into the impact of crowdsourcing for paid work on employability (Warwick Institute for Employment Research/Institute for Prospective Technological Studies (IPTS), Warwick und Sevilla 2013).

Guglielmo, Wayne J., Job sharing: flexibility has a price, in: Journal of Medical Economics 85/2 (2008) 40-43.

Håkansson, K., Språngbräda eller segmentering? En longitudinell studie av tidsbegränsat anställda (Springboard or segmentation? A longitudinal study of temporary employees) (IFAU - Institutet för Arbetmarknadspolitisk Utvärdering, Uppsala 2001).

Holtgrewe, U., New new technologies. The future and the present of work information and communication technology, in: New Technology, Work and Employment 29/1 (2014) 924.

Horton, J. J.; Chilton, L. B., The Labor Economics of Paid Crowdsourcing (= EC 10 Proceedings of the 11th ACM conference on Electronic commerce, Cambridge, MA, 2010).

ILO (International Labour Organisation), On-call work and „zero hours“ contracts (= Information Sheet No. WT-15, Genf 2004).

Inkson, K.; Heising, A.; Rousseau, D. M., The Interim manager: Prototype of the $21^{\text {st }}$-century worker?, in: Human Relations 54/3 (2001) 259-284.

Ipeirotis, P., Demographics of Mechanical Turk (= Working Paper CeDER 10-01, New York University, Stern School of Business, New York 2010).

Irani, L.; Silberman, S., Turkopticon: Interrupting Worker Invisibility in Amazon Mechanical Turk (Paris 2013).

Isidor, R.; Schwens, C.; Kabst, R., Interim Management Utilization, Firm Flexibility and its Impact on Firm Performance, in: International Journal of Human Resources Development and Management (2014).

Jas , P., The role of interim managers in performance improvement: evidence from English local authorities, in: Public Money \& Management 33/1 (2013) 15-22.

Karlsson, K., Bemanningsföretag \& Arbetsmarknad - Bemanningsföretagens uppkomst och utveckling i Sverige (= Master thesis, Luleå University, Luleå 2009).

Kittur, A.; et al., The Future of Crowd Work (= Stanford University, 16th ACM Conference on Computer Supported Coooperative Work, Stanford 2013). 
Klebe, T.; Neugebauer, J., Crowdsourcing: Für eine handvoll Dollar oder Workers of the crowd unite?, in: Arbeit und Recht 1 (2014) 4-7.

Layte, R.; O'Connell, P. J.; Russell, H., Temporary jobs in Ireland: does class influence job quality?, in: Economic and Social Review. 39 (2008) 81-104.

Leighton, P.; Brown, D., Future Working: the rise of Europe's Independent Professionals (iPROS) (European Forum of Independent Professionals (EFIP)/PCG, London 2013).

Maschke, M.; Nies, G.; Vogl, G., Mobile Arbeit: zwischen Autonomie und Fremdbestimmung, in: WSI-Mitteilungen 2 (2014) 156-159.

Papsdorf, C., Wenn Surfen zur Arbeit wird. Crowdsourcing im Web 2.0 (Frankfurt am Main 2009).

Pennycook, M.; Cory, G.; Lakeson, V., A Matter of Time: The rise of zero-hours contracts (London 2013).

Platman, K., Portfolio careers and the search for flexibility in later life, in: Work, Employment \& Society 18/3 (2014) 573-599.

Pollitt, D., Challenging, flexible - and out of the way of office politics, in: Human Resource Management International Digest 16/4 (2008) 30-32.

Popma, J., The Janus face of the „New Ways of Work“. Rise, risks and regulation of nomadic work (= ETUI Working Paper, Brüssel 2013).

Rubery, J.; Grimshaw, D.; Hebson, G., Zero hours in social care: policy brief (Manchester Fairness at Work Research Centre, Manchester 2014).

Schaffers, H.; et al., The Future Workspace. Mobile and Collaborative Working Perspectives (The MOSAIC Consortium, Telematica Instituut, Enschede 2006).

Silberman, S. M.; Irani, L.; Ross, J., Ethics and Tactics of Professional Crowdwork, in: XRDS 17/2 (2010) 39-43.

The Russam GMS, The UK Interim Management Market Snapshot Survey, December (London 2010).

Unionen, Jobbet alltid närvarande (Malmö 2013).

Vinnova, Användningsdriven utveckling av IT i arbetslivet, No. 2 (Stockholm 2007).

Vötsch, J.; Titsch, K., ArbeitgeberInnenzusammenschluss (AGZ) im landwirtschaftlichen Bereich (Präsentationsfolien, 2011).

Wheatley, Is it good to share? Debating patterns in availability and use of job share, Discussion papers in Economics (Nottigham Trent University 2013).

Wild, E., Time and Chance: Planning and Preparing for a Portfolio Career (Wild ReSearch, London 2012).

Wölfing, S.; Wünsche, G.; Hartmann, T., Arbeitgeberzusammenschlüsse in Brandenburg ein Beitrag zur Umsetzung des Flexicurity-Ansatzes (Ministerium für Arbeit, Soziales, Gesundheit und Familie, Berlin, 2007).

\section{Zusammenfassung}

Wenngleich die überwiegende Mehrheit der europäischen Beschäftigungsverhältnisse nach wie vor der traditionallen Standardbeschäftigung (unbefristeter Vollzeitarbeitsvertrag oder Selbstständigkeit) entspricht, ist eine steigende Heterogenität an Beschäftigungsformen zu beobachten. Dies bezieht sich entweder auf eine geänderte Arbeitgeber-Arbeitnehmer-Beziehung oder auf veränderte Arbeitsorganisation und -praktiken, oder eine Kombination dieser beiden Aspekte. Während manche der neuen oder an Bedeutung gewinnenden Beschäftigungstrends Vorteile sowohl für Arbeitgeber als auch ArbeitnehmerInnen mit sich bringen, sind andere hinsichtlich ihrer Auswirkungen auf die Arbeitsbedingungen und den Arbeitsmarkt bedenklich. 


\section{Abstract}

While the vast majority of European employment relationships is still traditional standard employment (indefinite full-time employment contract or self-employment), an increasing heterogenity of employment forms can be observed. This relates either to a changed relationship between employer and employee or to changed work organisation and work patterns, or a combination of both. Some of the new or increasingly important employment trends have advantages for both employers and workers, but others raise concerns regarding their implications for working conditions and the labour market.

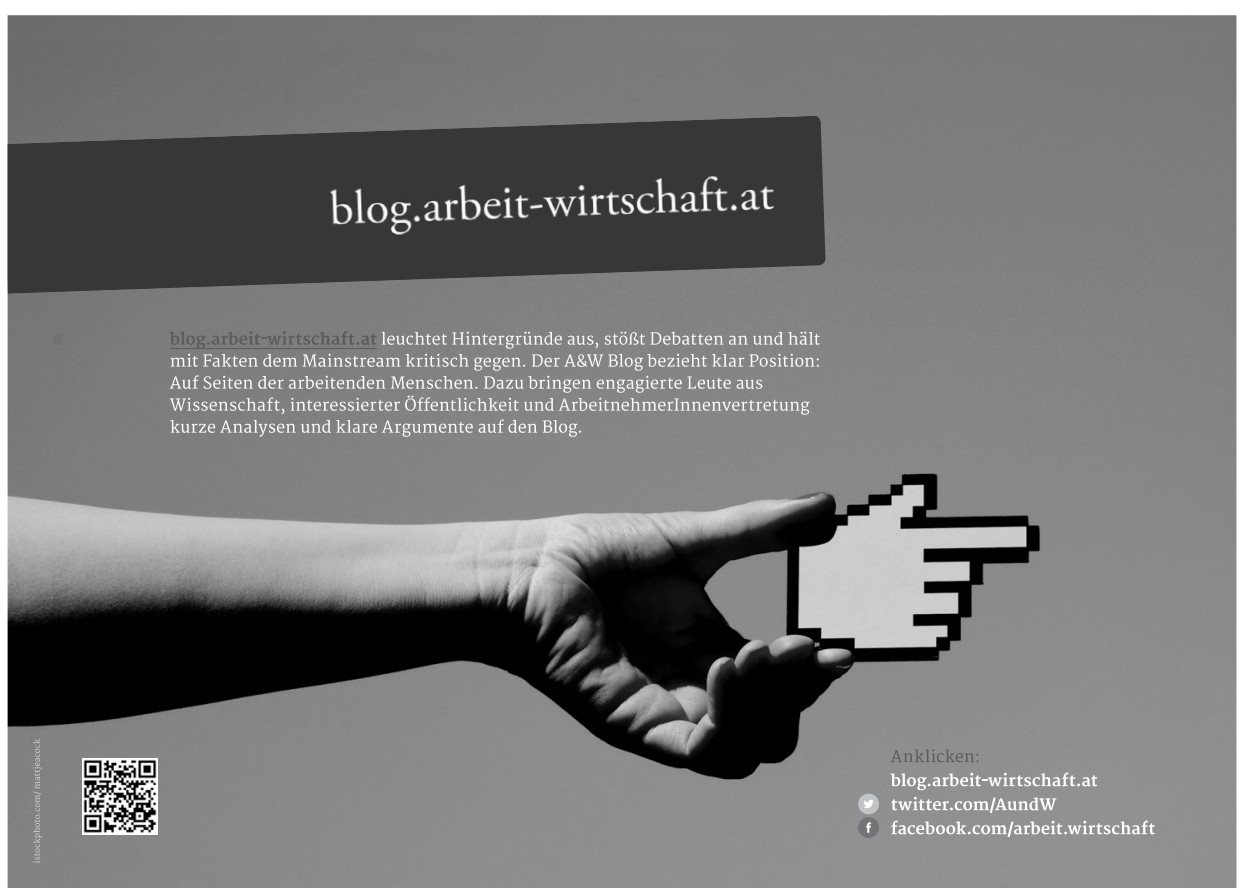

